\title{
Evaluation of the Knees of Asymptomatic Kangoo Jumpers with MR Imaging
}

\author{
Barış Polat ${ }^{1 *}$, Deniz Aydın², Ayşe Esin Polat ${ }^{3}$, Tahsin Gürpınar ${ }^{4}$, \\ Ramadan Özmanevra ${ }^{1}$, and Mehmet Alp Dirik ${ }^{5}$
}

\begin{abstract}
Purpose: The aim of this study is to determine the prevalence of pathologic findings in asymptomatic knees of Kangoo Jumpers by using a 3T MRI and to compare them with age and sex-matched controls who do not regularly participate in any impact sports.
\end{abstract}

Methods: Both knees of 18 Kangoo Jumpers were examined by 3T MRIs in a total of 36 MRI scans. The control group was comprised of 20 volunteers from the same age group and with similar weights who did not participate in any competitive sports, in a total of 40 MRI scans. Two orthopedists and one radiologist independently assessed all images for the presence or absence of any abnormalities.

Results: In 32 (88.9\%) of the 36 Kangoo Jumpers' knees, one or more abnormalities were observed. The most prevalent abnormality was bone marrow edema, which was detected in 32 knees (88.9\%). The other significant findings were quadriceps tendinopathy (80.6\%), patellar tendinopathy (63.9\%), gastrocnemius tendinopathy (63.9\%), infrapatellar fat pad edema (75\%), suprapatellar fat pad edema (63.9\%), meniscal signal change $(72.2 \%)$ and cartilage damage in the patellofemoral joint $(72.2 \%)$. There were no statistically significant differences in terms of joint effusion $(8.3 \%)$, ganglion cysts $(8.3 \%)$ or tibiofemoral joint cartilage injury $(0 \%)$.

Conclusion: This study reveals many types of knee MRI findings of asymptomatic Kangoo Jumpers compared to the control group. These MRI findings may be associated with acute knee injuries or chronic joint problems such as osteoarthritis, which may develop in long-term follow-up studies.

Keywords: bone marrow edema, Kangoo Jump, knee, magnetic resonance imaging

\section{Introduction}

Kangoo Jump (Kangoo Jumps, Sion, Switzerland) is a sport involving jumping that is performed with specially designed rebound shoes. Kangoo Jump is becoming increasingly popular and it can be used for jogging, athletic training, sports strengthening, and conditioning. The benefits of this

'Department of Orthopaedics and Traumatology, Kyrenia University, Dr. Suat Günsel Hospital, Şehit Yahya Bakır, Sokak Karakum, Kyrenia, Turkish Republic of North Cyprus

${ }^{2}$ Department of Orthopaedics and Traumatology, Near East University Hospital, Nicosia, Turkish Republic of North Cyprus

${ }^{3}$ Department of Orthopaedics and Traumatology, Dr. Akçiçek State Hospital, Kyrenia, Turkish Republic of North Cyprus

${ }^{4}$ Department of Orthopaedics and Traumatology, Istanbul Training and Research Hospital, Istanbul, Turkey

${ }^{5}$ Department of Radiology, Kyrenia University, Dr. Suat Günsel Hospital, Kyrenia, Turkish Republic of North Cyprus

*Corresponding author, Phone: +90-533-844-47-55, Fax: +90-392-815-99-98, E-mail: drbpolat@hotmail.com

(O2019 Japanese Society for Magnetic Resonance in Medicine

This work is licensed under a Creative Commons Attribution-NonCommercialNoDerivatives International License.

Received: August 6, 2018 | Accepted: December 19, 2018 sport include reduced body fat, tightening and strengthening muscles, increased agility and it provides aerobic exercise for the heart. Kangoo Jumps fitness programs offer an enjoyable form of physical activity to people of all ages. The rebound shoes used in Kangoo Jumps were primarily developed to reduce the impact stress on joggers' joints. ${ }^{1}$ Their balanced impact protection system reduces the impact force to the ground by up to $80 \%$. Conversely, jogging or jumping intensively with kangoo rebound shoes may lead to overuserelated pain and musculoskeletal injuries.

Kangoo Jumps is recommended as an effective plyometric exercise, particularly for competitive sports. Plyometric exercises are one of the most effective ways of increasing the vertical jump, speed, and strength of an athlete. These explosive movements allow the creation of maximum force in a short amount of time. The design of the rebound shoes incorporating ankle wraps reduces the risk of ankle injury as the pronation and supination are significantly reduced.

The knee joint is the most common cause of complaints in Kangoo Jumpers in our clinical practice. One of the two Kangoo Jumpers examined in the outpatient clinic had overuse-related pain caused by generalized bone marrow 
edema and the other had a grade 2 medial collateral ligament tear at a different time. After these injuries, we wanted to determine the possible pathologies by using 3T MRI in asymptomatic Kangoo Jumpers' knees.

MRI is the most reliable non-invasive diagnostic technique for the assessment of musculoskeletal lesions associated with sport practice, such as bone marrow edema (BME), joint effusion, as well as cartilage, meniscal, ligament and tendon lesions..$^{2-7}$

A range of positive MRI findings were detected in the asymptomatic knees of athletes from various sports, such as basketball, football, swimming and running. ${ }^{2,4,5,7}$ Positive radiological findings should be considered, although they are clinically asymptomatic. These imaging findings may guide the prevention of future irreversible functional disabilities. To our knowledge, there has been no such study published about the knee MRI findings of asymptomatic Kangoo Jumpers. We aimed to determine the prevalence of pathologic findings in the asymptomatic knees of Kangoo Jumpers by using $3 \mathrm{~T}$ MRI and to compare them with age and sexmatched controls who do not regularly participate in any impact sports.

\section{Materials and Methods}

\section{Patients}

This cross-sectional control study was conducted at Dr. Suat Günsel Kyrenia University Hospital between January 2018 and April 2018. We obtained ethical approval for this study from the Institutional Review Board of our hospital (Ref No: RY-2018-07). Athletes and individuals in the control group participated in the study voluntarily and signed informed consent forms. The demographic information of a total of 18 Kangoo Jumper candidates and a control group of 20 non-athletic candidates was obtained via the questionnaire designed for the study.

The inclusion criteria for the Kangoo Jumpers were: being asymptomatic related to the knees, regularly participating in a Kangoo Jumps fitness program held at a minimum frequency of three times a week for 1 hour/day and having participated in a Kangoo Jumps fitness program for at least 6 months. Exclusion criteria included previous surgery on the knee, hip or ankle; history of any traumatic injuries that led to breaks from the regularly Kangoo Jumps fitness program; osteoarticular diseases or lower limp malalignment; history of chronic disease and body mass index $(\mathrm{BMI})>30$.

The inclusion criteria for the control group were being asymptomatic related to the knees, with a corresponding age and weight range with the athletes. The exclusion criteria were the participate in any competitive sports more than once a month in the last 6 months; history of previous surgery on the lower limb or any traumatic knee lesion that led to breaks in daily activities; osteoarticular diseases or lower limp malalignment; history of chronic disease and BMI $>30$.
Ultimately, 18 Kangoo Jumper candidates and 20 nonsporting candidates were included in the study. Both knees of each participant were examined by 3T MRI, with 36 MRI scans in the Kangoo Jump group and 40 MRI scans in the control group.

\section{Magnetic resonance imaging}

All examinations were performed with a 3T MRI machine (Siemens, Magnetom, Skyra, Erlangen, Germany) and a 15-channel knee-dedicated Tx/Rx 15-channel coil. We used our standardized protocol which basically has three sequences in sagittal orientation; 1) fat-suppressed proton density weighted turbo spin-echo (FS PD TSE) sequence, (TR/TE, 3090/25), 2) $\mathrm{T}_{1}$-weighted $\left(\mathrm{T}_{1}\right)$ turbo spin-echo sequence, (TR/TE, 473/12), 3) $\mathrm{T}_{2}$ fat saturated water excitation double echo steady state-3D ( $\mathrm{T}_{2}$ FS 3D DESS WE) sequence, (TR/ TE, 1484/504), two sequence in coronal orientation; 1) fatsuppressed proton density weighted turbo spin-echo (FS PD TSE) sequence, (TR/TE, 2640/34), 2) $\mathrm{T}_{2}$ turbo inversion recovery magnitude short tau inversion recovery $\left(\mathrm{T}_{2}\right.$ TIRM COR STIR) sequence, (TR/TE, 4560/25) and one sequence in axial orientation; 1) fat-suppressed proton density weighted turbo spin-echo (PD_TSE_FS_TRA) sequence, (TR/TE, 4150/58)]. Additionally, FS PD TSE sequence scan time $83 \mathrm{~s}$. Bandwidth: $246 \mathrm{hz} / \mathrm{px} ., \mathrm{T}_{1} \mathrm{~W}$ TSE sequence scan time $163 \mathrm{~s}$. Bandwidth: $181 \mathrm{hz} / \mathrm{px} ., \mathrm{T}_{2}$ FS 3D DESS WE sequence scan time 241 s. Bandwidth: 222 hz/px., T 2 TIRM_COR sequence scan time $96 \mathrm{~s}$. Bandwidth: $246 \mathrm{hz} / \mathrm{px}$. The parameters used for image acquisition were a $320 \times 256$ matrix, $19 \times 17 \mathrm{~cm}$ dual FOV, $3.0 \mathrm{~mm}$ slice thickness with $0.8 \mathrm{~mm}$ interslice gap.

Knee MRI scans were evaluated for the presence or absence of the following findings which were described as abnormal when the following criteria were met.

1. Fat pad edema (suprapatellar, infrapatellar): Increased signal intensity in fat-suppressed $T_{1}$-weighted fast spin-echo $\left(T_{1} F S E\right)$, fat-suppressed $P D$ sequence and reduced signal intensity in $T_{1}$ FSE sequences.

2. Tendinopathy (quadriceps, patellar, gastrocnemius): Thickening or signal intensity changes in FSE, PD, and $\mathrm{T}_{1}$ FSE sequences.

3. Meniscal injury: Increase in signal intensity in FSE, $\mathrm{PD}$ and $\mathrm{T}_{1}$ FSE sequences that are graded as follows: Grade I, intrasubstance, globular signal change and does not reach the superior or inferior articular surface. Grade II, intrasubstance, linear signal change and does not reach the superior or inferior joint surface. Grade III, signal change reaches the superior or inferior articular surface or both., 4

4. Cartilage injury (tibiofemoral, patellofemoral joint): Increase in signal intensity in FSE, PD and $T_{1}$ FSE sequences that are graded as follows; Grade I, focal areas of hyperintensity with the normal contour of cartilage surface. Grade II, damage compromising $<50 \%$ of the thickness of the cartilage. Grade III, 
damage compromising $>50 \%$ of the thickness of the cartilage but not extended to the subchondral bone tissue. Grade IV exposed subchondral bone. ${ }^{6-8}$

5. BME (tibiofemoral, patellofemoral, femoral longitudinal): Increased signal intensity in FSE and PD sequence; and reduced signal intensity in $T_{1}$ FSE sequences. Any signal changes in the tibiofemoral or patellofemoral bone tissue were accepted as abnormal. In the sagittal sequence, greater than $1.5 \mathrm{~mm}$ thick signal change behind the anterior femoral cortex was considered abnormal.

6. Joint effusion: In the suprapatellar bursa in the midsagittal sequence, a quantity of sinovial fluid volume less than $5 \mathrm{~mm}$ was considered absent and a volume of fluid of $5 \mathrm{~mm}$ or more was considered abnormal. ${ }^{5}$

7. Ganglion cysts: Defined as present or absent in all three sequences in popliteal or parameniscal location.

\section{Analysis of the images}

A total of 76 knee MRIs were saved on a workstation for further analysis. Two orthopedists and one radiologist (BP, DA, and MAD) with 4, 6 and 8 years' experience in musculoskeletal imaging, respectively assessed all images independently. Findings were blinded to the study groups. The examiners were blind to the group of the subject. Where there was disagreement between the examiners, a reevaluation was made until a consensus was reached.

\section{Statistical analysis}

Mean, standard deviation, median lowest, highest, frequency and ratio values were used in the descriptive statistics of the data. The distribution of the variables was measured by the Kolmogorov-Smirnov test. The Mann-Whitney test was used in the analysis of quantitative independent data. A Chi-square test was used in the analysis of qualitative independent data. SPSS 22.0 software (SPSS Inc., Chicago, IL, USA) was used in the analyses. Statistical significance was considered to be present at $P<0.05$. Interobserver agreement was assessed using the kappa coefficient $(\kappa)$ statistical test. The $\kappa$-value between 0.8 and 1 was considered as perfect agreement.

\section{Results}

\section{Characteristics of the participants}

The study and control groups had similar baseline demographic characteristics which are shown in Table 1. Athletes who contributed to the study were selected randomly on a voluntary basis among those who regularly attended a Kangoo Jumps fitness program 3 days a week for 1 hour/day. The mean duration of the Kangoo Jumps fitness program was 14.4 months (range: 6-60).

\section{Abnormalities of the knees}

A total of 76 knee MRIs were analyzed, including 40 (53\%) candidates in the control group and 36 (47\%) Kangoo Jump athletes.

In the group of Kangoo Jumpers, 32 (88.9\%) of 36 knees had one or more abnormalities. The most prevalent abnormality was BME, which was detected in $32(88.9 \%)$ knees. Based on the regions where BME was seen, three groups were separated. The most common location of BME was immediately behind the anterior femoral cortex, called femoral longitudinal BME (88.9\%) (Fig. 1). The second most frequent area of BME was the patellofemoral joint which includes the patella and trochlea $(66.7 \%)$. The third most common site of BME was the tibiofemoral joint which includes the femoral condyles and tibial plateaus (47.2\%).

The second most common finding was quadriceps tendinopathy which was detected in 29 (80.6\%) knees. Both patellar and gastrocnemius tendinopathy were detected in 23 (63.9\%) knees. Infrapatellar and suprapatellar fat pad edema were detected in $27(75 \%)$ and $23(63.9 \%)$ knees, respectively (Fig. 2). The meniscal signal change was detected in $26(72.2 \%)$ knees. Signal change at the lateral meniscus was only detected in one knee with Grade I. Other signal changes were detected at the posterior horn of the medial meniscus in 22 knees with Grade I and in 3 knees with Grade II (Fig. 3). Articular cartilage damage was investigated in two different regions: patellofemoral and tibiofemoral. Cartilage damage in the patellofemoral joint which includes the patellar and trochlea articular surface was detected in $26(72.2 \%)$ knees

Table 1 Patients demographics

\begin{tabular}{|c|c|c|c|c|c|c|}
\hline \multirow[b]{3}{*}{ Age (years) } & \multicolumn{2}{|c|}{ Control group $(n=20)$} & \multicolumn{2}{|c|}{ Kangoo Jumpers $(n=18)$} & \multirow{2}{*}{\multicolumn{2}{|c|}{$P$-value }} \\
\hline & Mean $\pm \mathrm{SD}, n(\%)$ & Median & Mean $\pm \mathrm{SD}, n(\%)$ & Median & & \\
\hline & $28.8 \pm 3.9$ & 29.0 & $29.0 \pm 5.0$ & 28.5 & 0.825 & $\mathrm{~m}$ \\
\hline \multicolumn{7}{|l|}{ Sex } \\
\hline Female & $16(80.0)$ & & $15(83.3)$ & & \multirow{2}{*}{0.791} & \multirow{2}{*}{$\chi^{2}$} \\
\hline Male & $4(20.0)$ & & $3(16.7)$ & & & \\
\hline Height (cm) & $168.2 \pm 6.4$ & 167.5 & $167.5 \pm 7.6$ & 168.0 & 0.618 & $\mathrm{~m}$ \\
\hline Weight (kg) & $63.4 \pm 8.8$ & 60.5 & $65.2 \pm 10.6$ & 64.0 & 0.619 & $\mathrm{~m}$ \\
\hline Body mass index & $22.4 \pm 3.1$ & 21.4 & $23.2 \pm 2.7$ & 23.9 & 0.372 & $\mathrm{~m}$ \\
\hline
\end{tabular}

$\mathrm{m}$, Mann-Whitney $u$-test; $\chi^{2}$, Chi-square test. 


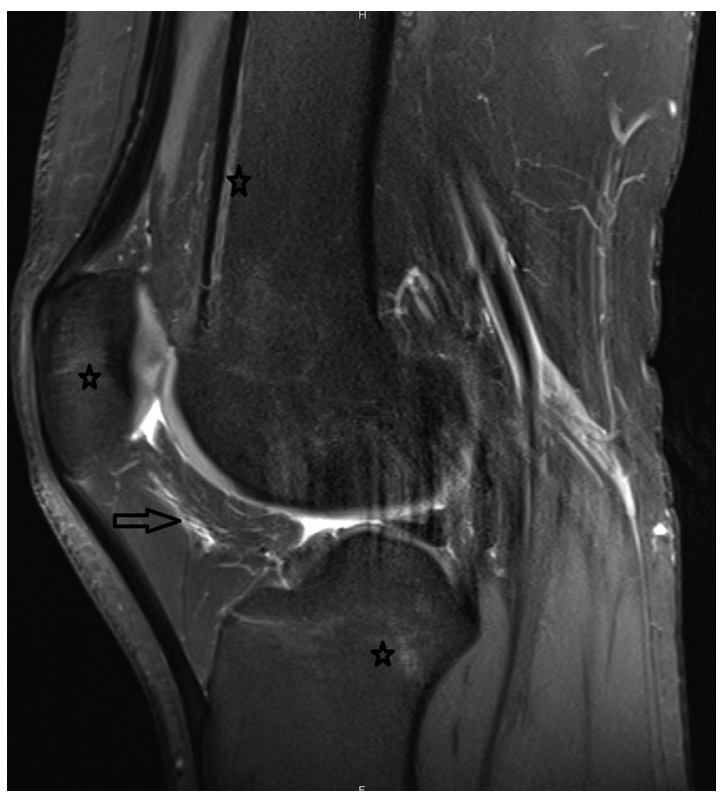

Fig. 1 27-year-old female asymptomatic Kangoo Jumper. Proximal asterisk shows femoral longitudinal bone marrow edema (BME), middle asterisk shows patellar BME, distal asterisk shows tibiofemoral joint BME. Arrow shows infrapatellar fat pad edema. Patellar and quadriceps tendinopathy are seen as signal change at their insertion.

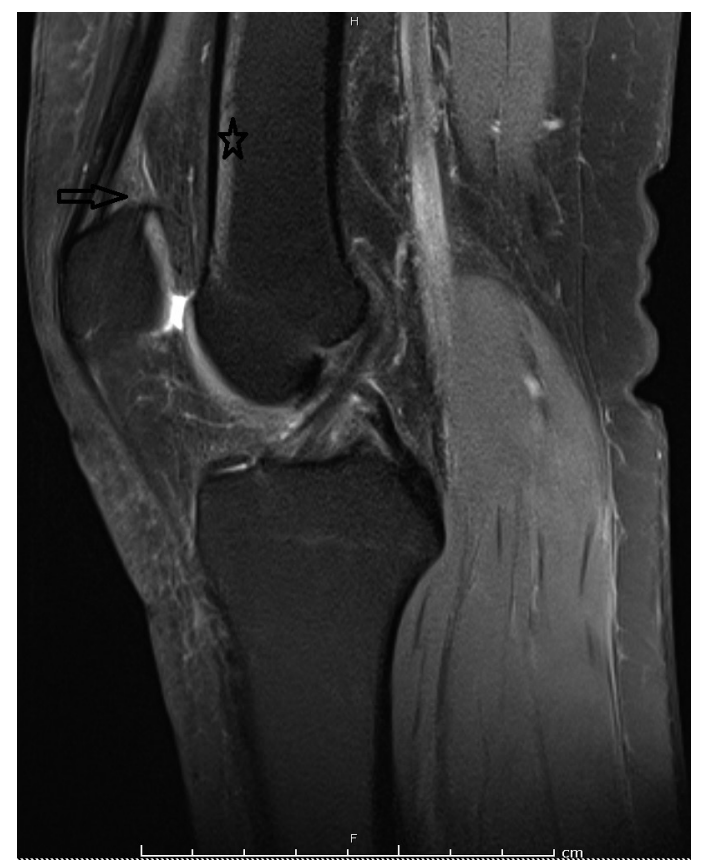

Fig. 2 29-year-old female asymptomatic Kangoo Jumper. Asterisk shows femoral longitudinal bone marrow edema (BME), arrow shows suprapatellar fat pad edema.

(Fig. 4). This cartilage damage was classified in 20 knees as Grade I and in six knees as Grade II. Cartilage damage in the tibiofemoral joint was not detected in any athletes. Joint effusion and ganglion cysts were detected in three different patients $(8.3 \%)$.
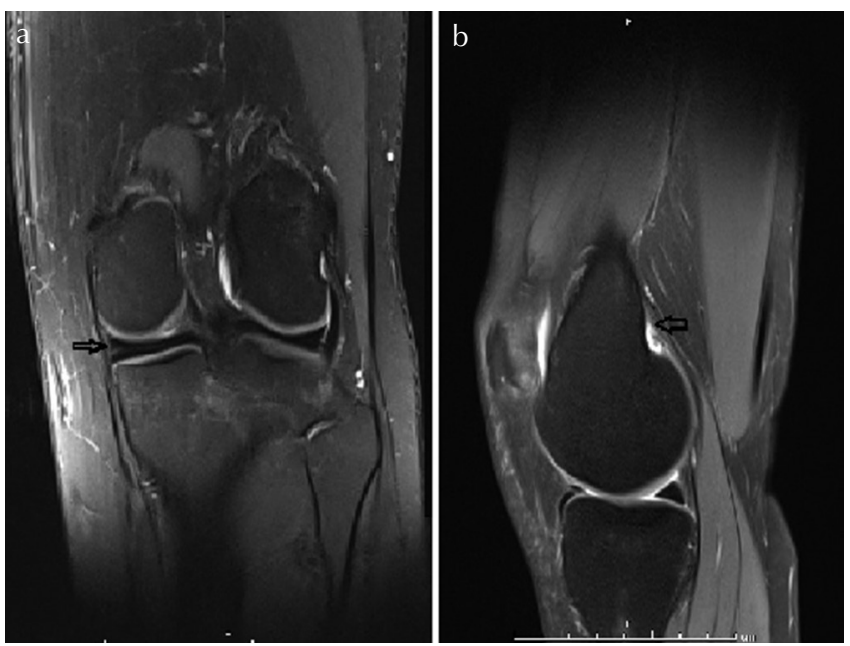

Fig. 3 31-year-old male asymptomatic Kangoo Jumper. (a) Arrow shows Grade I meniscal degeneration. (b) Arrow shows gastrocnemius tendinopathy.

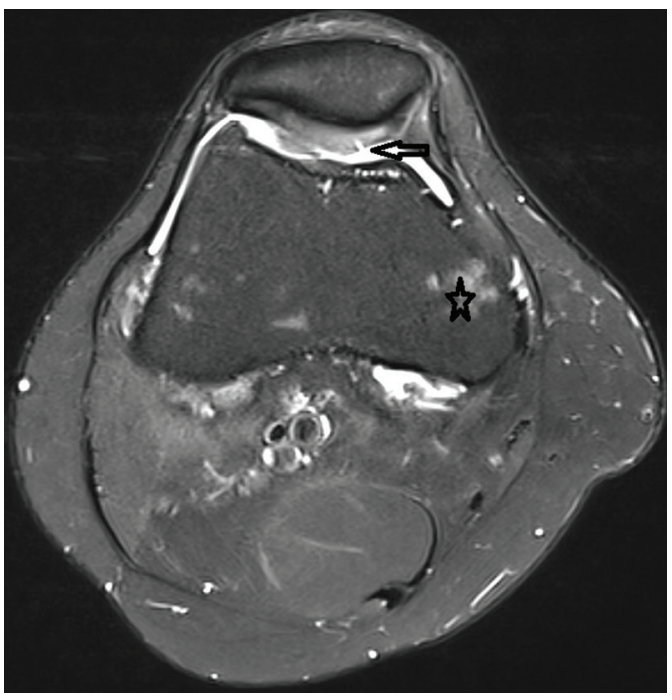

Fig. 4 33-year-old female asymptomatic Kangoo Jumper. Asterisk shows tibiofemoral bone marrow edema (BME), arrow shows tip II cartilage injury in the patellofemoral joint.

In the control group, 16 (40\%) of the 40 knees had one or more abnormalities. The most prevalent abnormality was infrapatellar fat pad edema which was detected in $11(27.5 \%)$ knees. In 9 (22.5\%) knees suprapatellar fat pad edema was detected, while quadriceps tendinopathy was found in $6(15 \%)$ knees, patellar tendinopathy in $5(12.5 \%)$ knees and gastrocnemius tendinopathy in $8(20 \%)$ knees. BME was detected in only $4(10 \%)$ knees. Patellofemoral joint BME was detected in $3(7.5 \%)$ knees and tibiofemoral joint BME was detected in 2 (5\%) knees. Femoral longitudinal BME was not detected in any knee and 4 (10\%) knees exhibited meniscal signal changes. All of these signal changes were Grade I at the medial meniscus posterior horn. A total of $8(20 \%)$ knees exhibited patellofemoral joint cartilage injury. 
All of these injuries were classified as Grade I. Tibiofemoral joint cartilage injury was not detected in any knee.

In the case group, the suprapatellar, infrapatellar fat pad edema, quadriceps, patellar, gastrocnemius tendinopathy, meniscal injury, patellofemoral joint cartilage injury, BME was significantly higher than in the control group $(P<0.05)$. Tibiofemoral joint cartilage injury, joint effusion and ganglion cysts in the case and control groups did not differ significantly $(P>0.05)$. Interobserver agreement $(\kappa)$ was 0.83 for all assessments. The results are summarized in Table 2 .

Table 2 Abnormalities detected in the knees of Kangoo Jumpers and controls

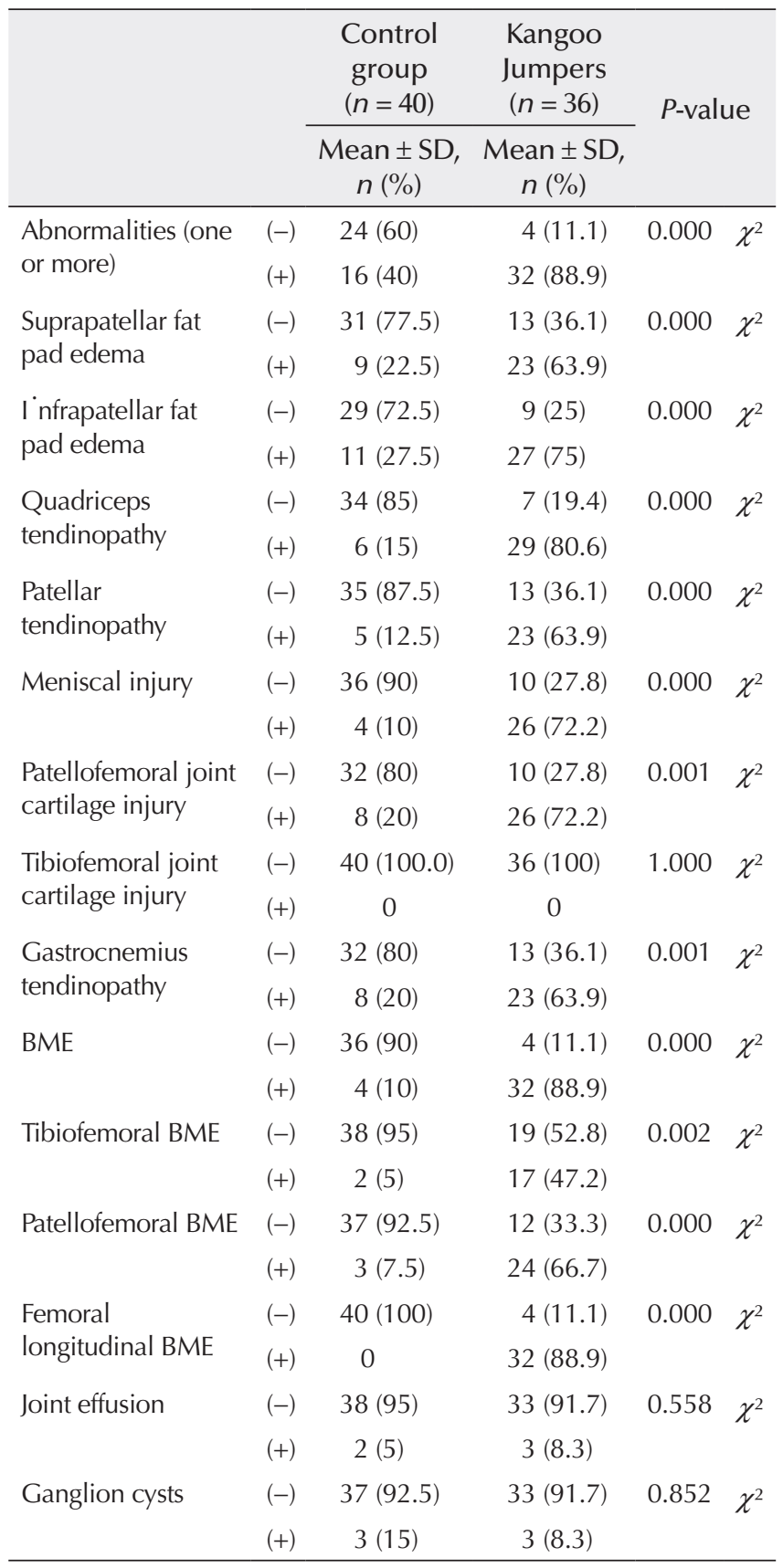

$\mathrm{BME}$, bone marrow edema; $\chi^{2}$, chi-square test.

\section{Discussion}

This study revealed significant differences in the knee MRI findings of asymptomatic Kangoo Jumpers compared to the control group. These findings will contribute to the assessment and interpretation of knee MRI findings in Kangoo Jumpers who undergo MRI to investigate any suspected trauma or osteoarticular lesion.

The most significant MRI finding in Kangoo Jumpers' knees was BME. Most traumatic knee BMEs correlate with the mechanism of the knee injury. Sanders classified traumatic knee BME into five different patterns, which are pivot shift, clip, dashboard, hyperextension injuries and lateral patellar dislocation., ${ }^{9,10}$ All these BME patterns appear after acute trauma and extend to the subchondral bone tissue. Several authors have shown an association between articular cartilage defects and subchondral BME. ${ }^{11,12}$ Not only acute trauma, but also the altered or increased mechanical stresses on the bone lead to BME. In $88.9 \%$ of the athletes, a femoral longitudinal BME was seen just posterior of the anterior femoral cortex. This femoral longitudinal BME does not extend to cartilage tissue, unlike traumatic BME. This BME does not cause any thickening of the periost or anterior femoral cortex and also does not cause edema in the surrounding soft tissue. Repetitive jumping can increase the tensile force over the anterior femoral cortex. The anterior femoral cortex exhibits respond to this mechanical stress. This response includes edema, microfractures and trabecular remodeling. This histological change is seen as a BME in MRI. ${ }^{5,6}$ Also, we detected $47.2 \%$ BME in femoral condyles or tibial plateaus. This rate is high in comparison to the control group and is compatible with $81 \%$ of asymptomatic basketball players, ${ }^{7} 50 \%$ and $41.3 \%$ of asymptomatic soccer players, ${ }^{5,6}$ and $26.9 \%$ of asymptomatic swimmers. ${ }^{2}$

Other significant findings in the Kangoo Jumpers included patellar tendinopathy (63.9\%), quadriceps tendinopathy (80.6\%) and gastrocnemius tendinopathy (63.9\%). Repetitive jumping increases the forces to the extensor tendons, particularly on landing. ${ }^{13}$ Straining in the tendons leads to microtears. These microtears heal improperly with type I collagen produced by tenocytes, which leads to tendon degeneration. ${ }^{14}$ Degeneration in these tendons could cause tenderness, painful swelling, restrictive sporting activity or can be detected incidentally in the athlete's knee MRI in the presymptomatic phase, as in our athletes. Patellar and quadriceps tendinopathy have been reported to occur frequently in sports involving recurrent jumping ${ }^{13}$. The study we conducted revealed that the ratio of patellar and quadriceps tendinopathy was compatible with a previous study in which basketball players' knees were evaluated with MRI. ${ }^{7}$

In this study, infrapatellar and suprapatellar fat pad edema were detected in Kangoo Jumpers' knees $75 \%$ and $63.9 \%$, respectively. There are two widely accepted theories for fat pad edema formation. The first theory is excessive knee hyperextension or rotation and repetitive microtrauma. 
It is thought that the fat pad squeezes between the patella and femoral condyles and leads to inflammation, and the latter possible patellar maltracking can increase this squeeze and inflammation. ${ }^{15,16}$ In the literature, infrapatellar fat pad edema was detected in asymptomatic adolescent soccer players, asymptomatic adolescent swimmers and asymptomatic collegiate basketball players $35.7 \%, 53.8 \%$, and $75 \%$, respectively. ${ }^{2,5,7}$ This prevalence is increasing in sports that have intense flexion, extension and rotation movements. It is inevitable that this ratio will be high for those participating in the sport of Kangoo Jumping.

In this study, the meniscal signal change was detected in $72.2 \%$ of the Kangoo Jumpers' knees. In the study conducted by Pappas et al., ${ }^{7}$ knee MRI evaluations of asymptomatic collegiate basketball players showed 50\% meniscal signal change in the preseason and $61 \%$ in the postseason. Walczak et al. ${ }^{17}$ reported meniscal signal changes in $54 \%$ of asymptomatic The National Basketball Association (NBA) players. These results are compatible to our study. The meniscal signal change could have occured by myxoid degeneration, microcyst formation, calcification and fragmentation of collagen fibers. However, Grade II degeneration could be caused by intrasubstance meniscal tears and increased vascularity. ${ }^{18}$ This degenerative change is due to the fact that the repetitive flexion, extension and rotation movements cause excessive mechanical stress on the meniscus tissue.

In this study, patellofemoral joint cartilage signal change and irregularity was observed in $72.2 \%$ of the Kangoo Jumpers' knees. It was Grade I in 20 knees and Grade II in 6 knees. This finding is seen as a consequence of increased mechanical stress in the patellofemoral joint. It has been shown that there is a change in collagen structure, interstitial water content and proteoglycan content in the articular cartilage after exercise. ${ }^{19}$ As cartilage is an avascular tissue, there is limited ability to recover after injury. This can lead to signal change in MRI. Although a high prevalence of cartilage damage was seen in the patellofemoral joint, it was observed that the tibiofemoral joint was spared.

It was observed that the asymptomatic Kangoo Jumpers had a high prevalence of knee MRI abnormalities. This high prevalence is related to repetitive stress over the knee. However, technical mistakes like single leg landing ${ }^{20}$ or spreading the legs more than shoulder width while landing should also be questioned as a cause of increased MRI finding prevalence. The long-term effects of this sport have not been reported in literature. Tendinopathy findings as well as cartilage and meniscal signal changes are estimated to cause faster degenerative changes in the knee joint. On the other hand, the body may accommodate the repetitive stress over the anterior femoral cortex, femoral condyles and tibial plateau. Longitudinal femoral BME is the most significant finding in Kangoo Jumpers' knees as it has not previously been reported. We estimate that the incidence of this kind of edema may decrease in long-term Kangoo Jumpers with remodeling and strengthening of the loaded regions.
The high prevalence of findings in this study may be a result of the usage of 3T MRI instead of $3 \mathrm{~T}$ or $1.5 \mathrm{~T}$ MRI. It has been reported that $3 \mathrm{~T}$ MRI has improved the visualization of anatomical structures as well as the diagnostic sensitivity, specificity, grading of cartilage injury and other pathology. ${ }^{21-23}$

\section{Conclusion}

This study revealed many types of knee MRI findings of asymptomatic Kangoo Jumpers compared to the control group. These MRI findings can predict and prevent possible acute knee injury or chronic joint problems like osteoarthritis. However, long-term follow-up cohort studies are needed to better understand the significance of these findings.

\section{Acknowledgments}

The authors would like to thank Prof. Dr. Nail Bölükbaşı (Chief of Staff at Kyrenia University, Dr. Suat Günsel Hospital) for allowing the MRI examination of candidates.

\section{Conflicts of Interest}

The authors declare that they have no conflicts of interest.

\section{References}

1. Vance J, Mercer JA. Impact forces during running in a novel spring boot. In: Proceedings of the 25th Annual Meeting of the American Society of Biomechanics, San Diego, 2001; 109.

2. Soder RB, Mizerkowski MD, Petkowicz R, Baldisserotto M. MRI of the knee in asymptomatic adolescent swimmers: a controlled study. Br J Sports Med 2012; 46:268-272.

3. Stahl R, Luke A, Ma CB, et al. Prevalence of pathologic findings in asymptomatic knees of marathon runners before and after a competition in comparison with physically active subjects: a 3.0 T magnetic resonance imaging study. Skeletal Radiol 2008; 37:627-638.

4. Schueller-Weidekamm C, Schueller G, Uffmann M, Bader $\mathrm{T}$. Incidence of chronic knee lesions in long-distance runners based on training level: findings at MRI. Eur J Radiol 2006; 58:286-293.

5. Soder RB, Simões JD, Soder JB, Baldisserotto M. MRI of the knee joint in asymptomatic adolescent soccer players: a controlled study. AJR Am J Roentgenol 2011; 196: W61-W65.

6. Matiotti SB, Soder RB, Becker RG, Santos FS, Baldisserotto M. MRI of the knees in asymptomatic adolescent soccer players: a case-control study. J Magn Reson Imaging 2017; 45:59-65.

7. Pappas GP, Vogelsong MA, Staroswiecki E, Gold GE, Safran MR. Magnetic resonance imaging of asymptomatic knees in collegiate basketball players: the effect of one season of play. Clin J Sport Med 2016; 26:483-489.

8. Noyes FR, Stabler CL. A system for grading articular cartilage lesions at arthroscopy. Am J Sports Med 1989; 17:505-513. 
9. Sanders TG, Medynski MA, Feller JF, Lawhorn KW. Bone contusion patterns of the knee at MR imaging: footprint of the mechanism of injury. Radiographics 2000; 20:S135S151.

10. Hayes CW, Brigido MK, Jarnadar DA, Propeck T. Mechanism-based pattern approach to classification of complex injuries of the knee depicted at MR imaging. Radiographics 2000; 20:S121-S134.

11. Kijowski R, Stanton P, Fine J, De Smet A. Subchondral bone marrow edema in patients with degeneration of the articular cartilage of the knee joint. Radiology 2006; 238:943-949.

12. Rubin DA, Harner CD, Costello JM. Treatable chondral injuries in the knee: frequency of associated focal subchondral edema. AJR Am J Roentgenol 2000; 174:1099-1106.

13. Lian O, Engebretsen L, Bahr R. Prevalence of jumper's knee among elite athletes from different sports: a crosssectional study. Am J Sports Med 2005; 33:561-567.

14. Khan KM, Maffulli N, Coleman BD, Cook JL, Taunton JE. Patellar tendinopathy: some aspects of basic science and clinical management. Br J Sports Med 1998; 32:346-355.

15. Saddik D, McNally EG, Richardson M. MRI of Hoffa's fat pad. Skeletal Radiol 2004; 33:433-444.

16. LLopis E, Padrón M. Anterior knee pain. Eur J Radiol 2007; 62:27-43.
17. Walczak BE, McCulloch PC, Kang RW, Zelazny A, Tedeschi $\mathrm{F}$, Cole BJ. Abnormal findings on knee magnetic resonance imaging in asymptomatic NBA players. J Knee Surg 2008; $21: 27-33$

18. Li CA, Kim MK, Kim IH, Lee JH, Jang KY, Lee SY. Correlation of histological examination of meniscus with MR images: focused on high signal intensity of the meniscus not caused by definite meniscal tear and impact on MR diagnosis of tears. Korean J Radiol 2013; 14:935-945.

19. Eckstein F, Hudelmaier M, Putz R. The effects of exercise on human articular cartilage. J Anat 2006; 208:491512.

20. Ugalde V, Brockman C, Bailowitz Z, Pollard CD. Single leg squat test and its relationship to dynamic knee valgus and injury risk screening. PM R 2015; 7:229-235.

21. Magee T, Williams D. 3.0-T MRI of meniscal tears. AJR Am J Roentgenol 2006; 187:371-375.

22. Kijowski R, Blankenbaker DG, Davis KW, Shinki K, Kaplan LD, De Smet AA. Comparison of 1.5- and 3.0-T MR imaging for evaluating the articular cartilage of the knee joint. Radiology 2009; 250:839-848.

23. Wong S, Steinbach L, Zhao J, Stehling C, Ma CB, Link TM. Comparative study of imaging at 3.0 $\mathrm{T}$ versus $1.5 \mathrm{~T}$ of the knee. Skeletal Radiol 2009; 38:761-769. 